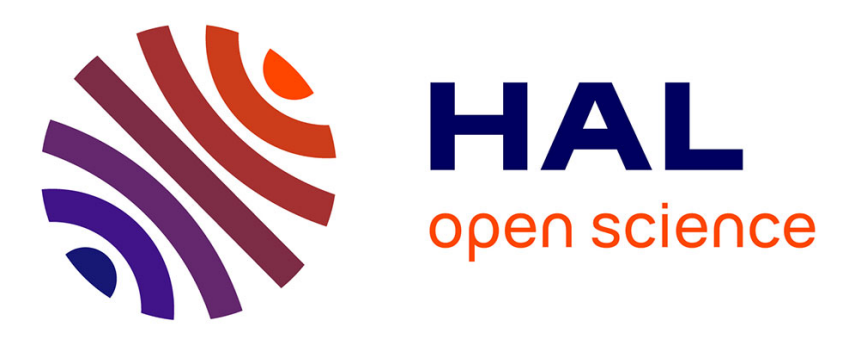

\title{
Diffuse Field Cross-Correlation in a Reverberation Chamber
}

Meriem Tamart, Francois Sarrazin, Elodie Richalot, Matthieu Davy, Julien de Rosny

\section{- To cite this version:}

Meriem Tamart, Francois Sarrazin, Elodie Richalot, Matthieu Davy, Julien de Rosny. Diffuse Field Cross-Correlation in a Reverberation Chamber. 2020 IEEE International Symposium on Antennas and Propagation \& USNC/URSI National Radio Science Meeting, Jul 2020, Montréal, Canada. 10.1109/IEEECONF35879.2020.9329710 . hal-03113188

\section{HAL Id: hal-03113188 \\ https://hal.science/hal-03113188}

Submitted on 18 Jan 2021

HAL is a multi-disciplinary open access archive for the deposit and dissemination of scientific research documents, whether they are published or not. The documents may come from teaching and research institutions in France or abroad, or from public or private research centers.
L'archive ouverte pluridisciplinaire HAL, est destinée au dépôt et à la diffusion de documents scientifiques de niveau recherche, publiés ou non, émanant des établissements d'enseignement et de recherche français ou étrangers, des laboratoires publics ou privés. 


\section{Diffuse Field Cross-Correlation in a Reverberantion Chamber}

\author{
M. Tamart, F. Sarrazin, E. Richalot \\ Université Paris-Est, ESYCOM (UMR9007), \\ UPEMLV, ESIEE-Paris, CNAM \\ 77454 Marne-la-Vallée, France \\ meriem.tamart@u-pem.fr
}

\author{
Matthieu Davy ${ }^{1}$, J. de Rosny ${ }^{2}$ \\ ${ }^{1}$ Univ Rennes, INSA Rennes, CNRS, IETR - UMR 6164 \\ F-35000 Rennes, France \\ ${ }^{2}$ ESPCI ParisTech, PSL Research University, CNRS, Institut Langevin \\ F-75005, Paris, France \\ 1 matthieu.davy@univ-rennes1.fr, ${ }^{2}$ julien.derosny@espci.fr
}

\begin{abstract}
The cross-correlation between diffuse field at two receiving antennas is very nearly equal to the direct response of one antenna to an impulse applied at the other one. Exploiting the diffuse wave field generated by rotation of a mechanical mode stirrer inside a reverberation chamber $(\mathrm{RC})$ excited by a single transmitting antenna, we outline the convergence of the cross-correlation toward the impulse response between the two receiving antennas. The laboratory demonstration shows that it is possible to characterize passively the coupling between two antennas in an RC.
\end{abstract}

\section{INTRODUCTION}

With technological advancement and the use of numerous integrated antennas, research on novel antenna characterization techniques has become a real need. We operate here the technique of diffuse-field cross-correlation to passively characterize the coupling between two antennas. This method was developed in different areas of theoretical and experimental physics and it has been applied to all types of waves (seismic [1], acoustic [2], elastic [3] and electromagnetic [4]). The use of this technique requires the reception of a diffuse field, meaning that the field received at location is homogeneous and isotropic. This diffuse field can be obtained either by a particular distribution of sources, or by diffusers introduced into the medium (multiple diffusion), or then quite simply by the reverberant nature of the medium.

The work presented here takes advantage of this technique to passively characterize the coupling between two antennas by noise correlation in a Reverberation Chamber (RC). We exploit the properties of the RC to generate a diffuse field using a single source and a mechanical mode stirrer. Averaging over different realizations, the reconstructed signal is expected to converge towards the impulse response between two receiving antennas.

\section{RESUlts}

\section{A. Experimental Setup}

The experiment was made in the RC of the ESYCOM laboratory $(2.751 \mathrm{~m} \times 2.951 \mathrm{~m} \times 2.354 \mathrm{~m}$, LUF around 400 $\mathrm{MHz}$ ) as depicted in Fig. 1. The two antennas under test (AUT) are two horn antennas ( $\mathrm{a}$ and $\mathrm{b}$ ) facing each other and separated with a distance of $45 \mathrm{~cm}$. They are placed inside the working volume of the RC and they are used in receiving mode. The source antenna (c) is directed to a mechanical mode stirrer in order to generate a diffuse field. The measurements are carried out at 100001 frequency points uniformly spaced on the frequency band of [1-8] GHz for 120 regularly space stirrer positions over a whole rotation, and two different tilts of the excitation antenna are considered (namely of Xdeg and Ydeg in regard to the horizontal plane).

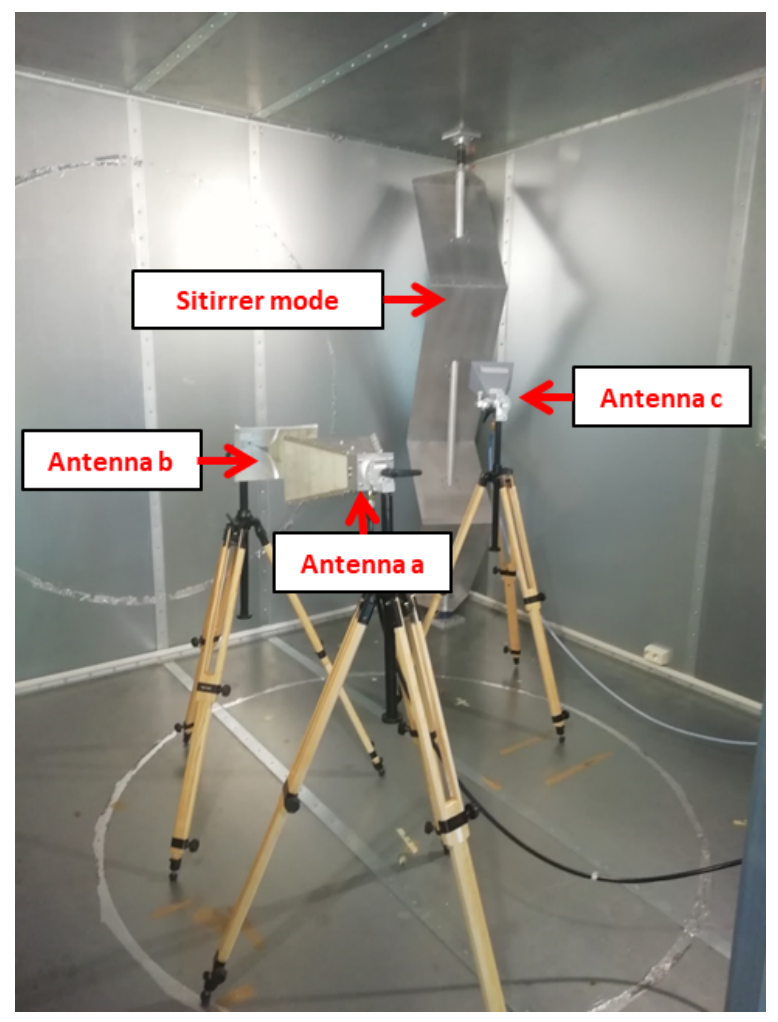

Fig. 1. Experimental setup in ESYCOM laboratory's RC.

\section{B. Data Processing}

Once the measurement data have been recovered, they are processed in the frequency domain because the calculation of the frequency correlation function is faster. First, we eliminate the amplitude information from the $Z_{i j}$ parameters by working only with the information contained in the phase $\Phi_{i j}$ of 
the signals. This allows for equivalent contributions from all frequencies in the working band. The effectiveness of phase information has already been demonstrated in [5].

Let $Z^{\prime}$ in (1) be the new parameters taking into account only the phase information, the indices $\mathrm{a}$ and $\mathrm{b}$ for the receiving antennas, and $\mathrm{c}$ for the source antenna. The $C_{a b}$ crosscorrelation is then calculated as in (2). The cross-correlation function is then converted in the time domain using an inverse Fourier transformation and it is averaged over the different stirring positions and excitation antenna orientations.

$$
Z_{i j}^{\prime}(f)=\exp \left(j \cdot \Phi_{i j}\right)
$$

and :

$$
C_{a b}(f)=Z_{a c}^{\prime} Z_{b c}^{\prime *}
$$

Figure 2 displays the reconstructed impulse response obtained by calculating the correlation function (red curve) and by direct measurement of the impulse responses between the antennas $a$ and $b$ (blue dash-dotted curve for the causal response and black dotted curve for the anti-causal one). As expected, the results obtained are well superimposed and the inter-correlation function converges towards the average impulse response between the antennas so that we can recover the ballistic wave and the first echoes in the RC.

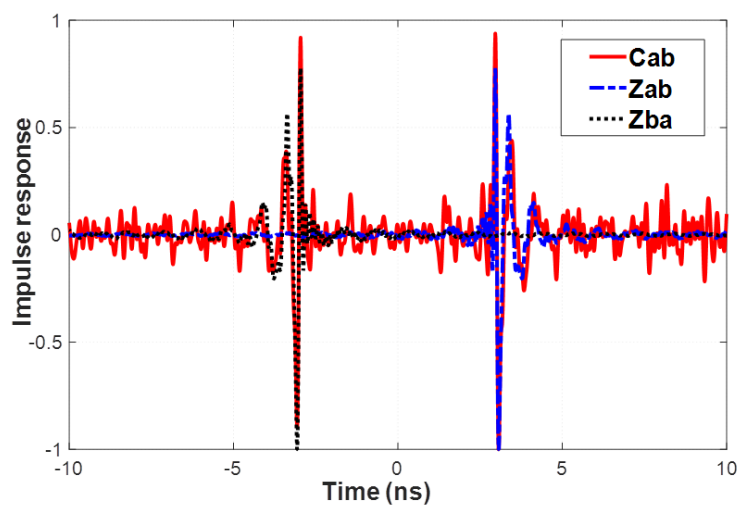

Fig. 2. Calculated cross-correlation function (red) and measured impulse responses (blue and black).

\section{Convergence Analysis}

Now, we study the reconstruction quality. The similarity of the reconstructed impulse response $C_{a b}$ calculated for different realizations with the reference impulse response obtaind with 120 stirer positioins, $C_{a b}$ is evaluated via the Pearson correlation coefficient $S$ (3) as a function of the number of available RC realizations. The results displayed in Fig. 3 shows that the correlation results obtained with 60 positions and 120 stirring positions are close, which means that in this case 60 $\mathrm{RC}$ realizations are sufficient to converge towards the impulse response between the two AUT.

$$
S=R e \frac{\int_{f} C_{a b}(f) \tilde{C}_{a b}^{*}(f)}{\left.\sqrt{(} \int_{f}\left|C_{a b}(f)\right|^{2} d f\right)\left(\int_{f}\left|\tilde{C}_{a b}(f)\right|^{2} d f\right)}
$$

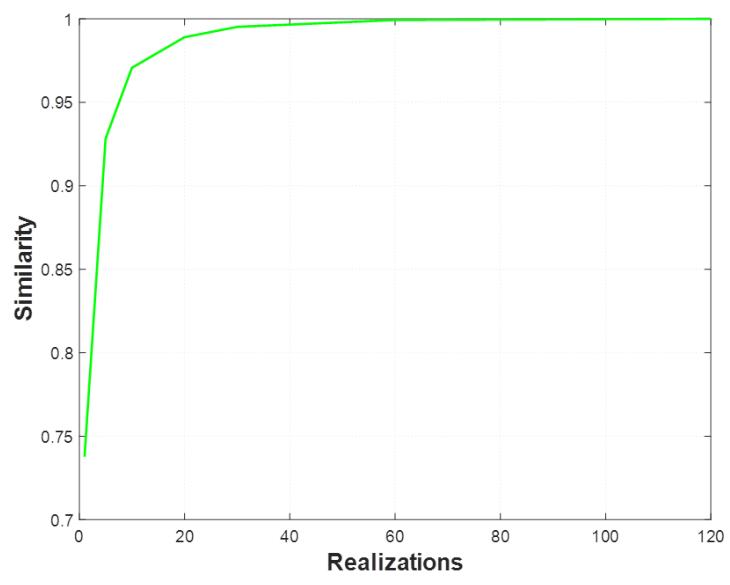

Fig. 3. Similarity Coefficient as a function of the number of equally spaced stirrer positions over a whole rotation

\section{CONCLUSION}

In this communication, we have used the diffuse field cross-correlation technique to passively estimate the impulse response between two antennas using a single source located in an RC. The experimental validation of this technique was exposed through measurements in the RC of the ESYCOM laboratory with two receiving horn antennas. We have presented the measurement results obtained to validate the principle of reconstruction of the impulse response by studying the influence of the number of realizations on the convergence of the field correlation towards the impulse response between the antennas. In a perspective, this work will be deepened to use the technique of cross-correlation in order to characterize the coupling between two antennas in reception which do not have the possibility of being used in transmission.

\section{REFERENCES}

[1] M. Campillo and A. Paul, "Long-Range Correlations in the Diffuse Seismic Coda", Science 299, 547 (2003).

[2] P. Roux, W. Kuperman, and N. Grp, "Extracting coherent wave fronts from acoustic ambient noise in the ocean", J. Acoust. Soc. Am. 116, 1995 (2004).

[3] L. Chehami, J. D. Rosny, C. Prada, E. Moulin, and J. Assaad, "E xtraction des fonctions de Green par corrélation de bruit : application pour la détection et l'imagerie de défaut dans des plaques minces réverbérante", Lebanese Science Journal, Vol. 16, Special Issue, 2015.

[4] M. Davy, J. de Rosny, and P. Besnier, "Green's FunctionRetrievalwithAbsorbingProbesinReverberating Cavities”, Phys. Rev. Lett., p.5, 2016.

[5] A. Derode, A. Tourin, and M. Fink, "Ultrasonic pulse compression with one-bit time reversal through multiple scattering", J. Appl. Phys., vol. 85, p. 6434, 2014. 